\title{
DETERMINAÇÃO DO SEXO DE PUPAS DO BICHO DA SEDA (BOMBYX MORI) POR MINERAÇÃO DE DADOS
}

\author{
Mario Mollo Neto ${ }^{\prime}$, Thomas Hideki Duarte Murakami².
}

1- $\quad$ Prof. Dr. da FCE da Universidade Estadual Paulista Júlio de Mesquita Filho, UNESP, Campus de Tupã/SP - Brasil. mariomollo@tupa.unesp.br

2- $\quad$ Aluno do Curso de Engenharia de Biossistemas da FCE da Universidade

Estadual Paulista Júlio de Mesquita Filho, UNESP, Campus de Tupã/SP - Brasil

Recebido em: 02/10/2017 - Aprovado em: 21/11/2017 - Publicado em: 05/12/2017 DOI: 10.18677/EnciBio_2017B80

\begin{abstract}
RESUMO
Um dos processos importantes na indústria de sericicultura que permite a separação dos indivíduos que poderão produzir fios mais longos e de melhor qualidade é a identificação do sexo do bicho da seda. Este artigo visa à construção de um protótipo de um sistema de penetração ótica, com infravermelho que permita inferir a existência, ou não, das glândulas de quitina. Objetiva-se realizar a inspeção visual no segmento do abdômen da pupa do bicho da seda através de emissão de infravermelho proveniente de um conjunto de leds e coletar a diferença das intensidades de entrada e saída das ondas emitidas por meio de foto transistores, e, de posse do resultado da diferença serão realizadas análises por mineração de dados com o software WEKA na modalidade de classificação, que permita inferir o gênero das pupas. Esta abordagem é rentável, de baixo custo, com boa velocidade de coleta e processamento, não sendo destrutiva para as pupas, diferentemente das técnicas de análise de DNA aplicadas atualmente. Os resultados apontam para a classificação por meio de uma árvore de decisão com grande precisão, que apresenta acertos de $96,75 \%$ o que é muito positivo para o processo produtivo que sofre com a classificação visual com índice de acerto menor que o alcançado nesta pesquisa.
\end{abstract}

PALAVRAS- CHAVE: Bombyx mori, Infravermelho, Mineração de dados.

\section{SILKWORM (BOMBYX MORI) PUPA SEX IDENTIFICATION WITH DATA MINING}

\begin{abstract}
One of the important processes in the sericulture industry that allows the separation of individuals who can produce longer wires and better is the identification of the sex of the silkworm. This article aims to build a prototype of an optical penetration system with infrared that allows to infer the existence or not of chitin glands. The aim is to carry out visual inspection on the segment of the silk pupae pupae through infrared emission from a set of leds and to collect the difference of the input and output intensities of the waves emitted by means of phototransistors, the results of the difference will be performed by data mining with WEKA software in the classification mode, which allows inferring the genus of the pupae. This approach is cost-effective, low cost, with good collection and processing speed, and is not destructive to pupae, ENCICLOPÉDIA BIOSFERA, Centro Científico Conhecer - Goiânia, v.14 n.26; p.938 2017
\end{abstract}


unlike currently applied DNA analysis techniques. The results point to the classification by means of a decision tree with great precision, which shows hits of $96.75 \%$ which is very positive for the productive process that suffers with the visual classification with a lower hit rate than that reached in this research.

KEYWORDS: Bombyx mori, Data mining, Infrared.

\section{INTRODUÇÃO}

A Agricultura de Precisão (AP) compreende um conjunto de técnicas e metodologias que visam otimizar o manejo das culturas e a utilização dos insumos agropecuários, proporcionando máxima eficiência econômica. As ferramentas de AP permitem o uso racional dos corretivos, fertilizantes e agrotóxicos garantindo a redução dos impactos ambientais decorrentes da atividade agropecuária (BRASIL, 2011).

Agricultura de Precisão, pode ser definida como a aplicação da TI em conjunto com a tecnologia de sensores envolvendo o uso de tecnologia eletrônica para coletar grande quantidade de dados no campo para uso no manejo da cultura específica e em um determinado local físico, como por exemplo, se aplica ao trabalho de Duhan et al. (2017) que traz os novos Biosensores para aplicação a monitoramento no campo.

Os principais problemas na execução do AP incluem a interpretação dos dados coletados, para a compreensão e análise dos mesmos. É necessário avaliar o ambiente, a cultura específica e as causas da variabilidade dos dados. O sistema utilizado deve ser capaz de propor estratégias sólidas para a gestão da variabilidade de todo o processo produtivo (MURAKAMI et al., 2007). A determinação do sexo de pupas por meio da penetração de infravermelho está sendo experimentada no exterior com grande sucesso, como pode ser visto nos trabalhos de Kamtongdee et al. (2013) e Sumriddetchkajorn et al. (2015).

De acordo com Fisher e Kebede (2011), há muitas vantagens no desenvolvimento de circuitos baseados em microcontroladores incorporando novas tecnologias de sensores em aplicações agrícolas. Microcontroladores e sensores de estado sólido podem ser encontrados em muitas aplicações industriais, comerciais e de consumo. Muitos sensores e componentes auxiliares (chips de memória, relógios, etc.) são projetados para interagir diretamente com os microcontroladores, simplificando o projeto de circuitos e sua modificação/customização. Uma variedade de linguagens de programação, de alto nível, também estão disponíveis, permitindo ao programador acessar recursos sofisticados e complexos e criar aplicativos. Os componentes são baratos, e podem ser obtidos na maioria das partes do mundo, por meio de um grande número de fornecedores.

A escolha correta destes sensores é importante, porque na realidade, a coleta de dados precisos não é fácil. Este fato pode ser visto com muito destaque no trabalho de Ojha et al. (2015) em que os autores dizem que estes dispositivos estão ligados à necessidade de automação e tomada de decisão inteligente, que é cada vez mais importante para realizar esta missão de coleta de dados para o monitoramento em tempo real.

O processamento dados provenientes de sensores é outra tecnologia que avança a passos largos. O uso de sistemas específicos para a aquisição e processamento de dados em tempo real permite aos pesquisadores, por exemplo, a detecção de variáveis importantes para determinados processos de criação do agronegócio, de uma forma não intrusiva, e observar e avaliar os eventos 
específicos com nenhum ser humano interferindo diretamente (MOLLO NETO, 2007). Atualmente essas ferramentas fazem parte da realidade no campo, nas criações, no processamento, armazenamento e distribuição da agricultura competitiva mundial. Isto pode ser visto no trabalho de Rehman et al. (2014), que afirmam que estes dispositivos da AP dão todo o suporte necessário ao sucesso dos empreendimentos.

A possibilidade de trabalhar com técnicas não intrusivas como as propostas nesta pesquisa, auxiliam na redução da complexidade, redução do tempo necessário para a determinação do sexo e reduzem o impacto sobre as pupas, evitando-se a pesquisa que deve ser desenvolvida para a análise de DNA, como pode ser visto nos trabalhos de Gan et al. (2012) e Tang et al. (2017).

A ferramenta padrão adotada para as análises da pesquisa é o software de Mineração de Dados WEKA, do Machine Learning Group da University of Waikato, como adotado na pesquisa de Naik e Samant (2016) com a aplicação de classificação de dados. Com a coleta de dados do sensor ótico proposto neste projeto, serão gerados bancos de dados extensos e, os mesmos serão classificados com a aplicação da ferramenta de mineração. Esta permitirá a geração das árvores de decisão para a construção dos algoritmos de controle do módulo proposto para a devida automatização do processo de detecção das glândulas das pupas. Esta nova área de pesquisa surgiu como um meio de extrair padrões ocultos ou informações implícitas previamente desconhecidas de grandes repositórios de dados (REZENDE et al., 2005). O fascínio com a promessa de análise de grandes volumes de dados levou a um número crescente de aplicações bem sucedidas de mineração de dados nos últimos anos.

Objetivou-se, neste trabalho avaliar a técnica de manejo não intrusivo para a detecção do sexo de pupas do bicho da seda com a construção de um protótipo de um sensor ótico montado em um gabinete portátil, para ser aplicado como instrumento indicador da existência de glândulas de quitina por meio da avaliação de penetração de ondas infravermelhas no abdômen das pupas que tenha baixo custo e boa precisão e velocidade na discriminação do sexo de cada indivíduo testado.

\section{MATERIAL E MÉTODOS}

Para o desenvolvimento desta pesquisa, os trabalhos foram norteados, inicialmente, com uma prospecção bibliográfica em bases nacionais e internacionais de periódicos, das quais foram obtidos os dados de fundamentação para os estudos. A seguir, foi construído o hardware para um protótipo com microprocessador, em plataforma arduino, com a conexão a um conjunto (RX/TX - transmissor/receptor) composto de um led infravermelho montado em uma base plataforma que permitiu acomodar as pupas sem causar desconforto ou ferimentos, e em contraposição à plataforma, foi montado um foto transistor que realizou a coleta das ondas infravermelhas que atravessaram as pupas.

Foram registrados os dados correspondentes às diferenças das intensidades obtidas das pupas de amostra (40 pupas previamente identificadas por especialistas com sexos conhecidos separadas em dois grupos sendo, 20 do sexo masculino, ou "macho" - grupo 1 - e 20 do sexo feminino, ou "fêmea" - grupo 2). Foram realizadas 10 repetições de medidas dos valores de infravermelho que atravessaram as pupas, totalizando 400 instâncias de amostra. Os resultados obtidos foram armazenados em um banco de dados para classificação. Este trabalho utilizou métodos apresentados no trabalho de Martín-Ramos et al. (2017). 
O banco de dados foi analisado por meio da classificação em ferramenta para mineração de dados com aplicação do algorítmo J48, como no trabalho de Yadav, e Chandel (2015) e, foram construídas as árvores de decisão que permitiram a inferência da existência, ou não, das glândulas de quitina, determinando o sexo de cada uma das pupas analisadas.

\section{RESULTADOS E DISCUSSÃO}

\section{Resultados}

A construção do protótipo baseado em microcontrolador arduino foi construído como apresenta a Figura 1, onde tem-se a vista do módulo com as ligações com a plataforma que tem embutidos em sí o Led infravermelho, gerador do feixe principal de infravermelho e o fototransistor de captação da intensidade do feixe que atravessa a pupa. Na Figura 1, é possível observar a pupa inserida na plataforma de análise.

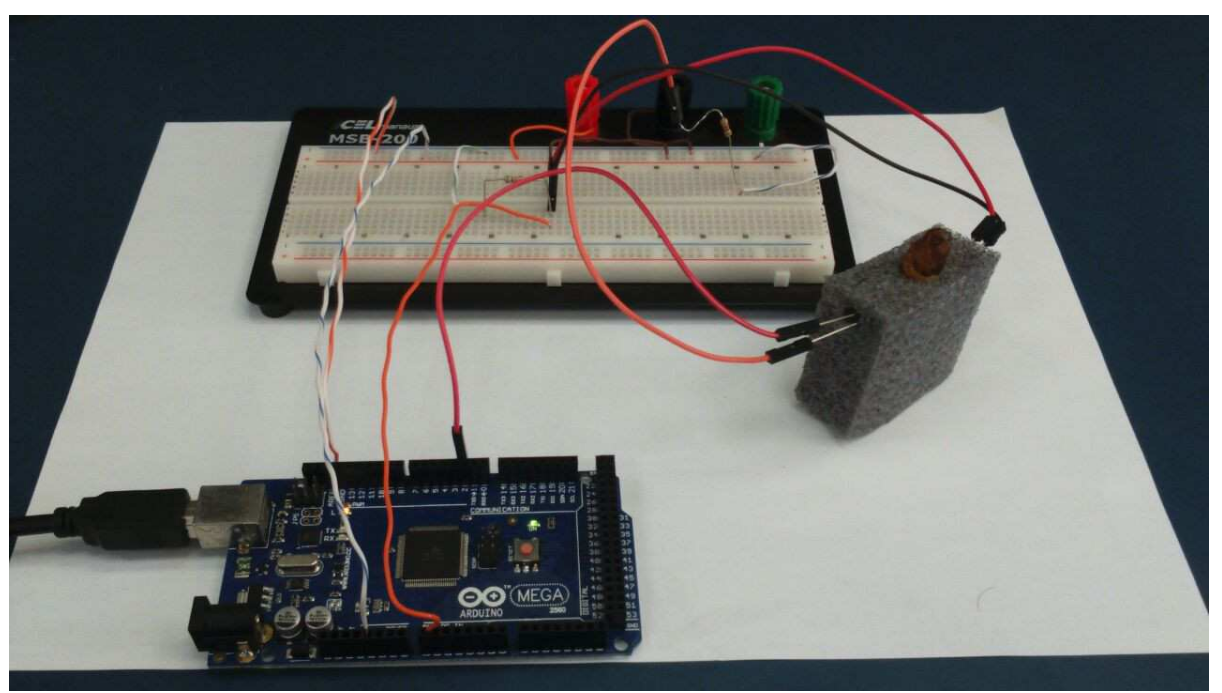

FIGURA 1. Módulo protótipo microprocessado com plataforma arduino montado para a experimentação de avaliação de sexo das pupas do bicho da seda. Fonte: Os autores.

Todo o circuito foi alimentado pela porta USB do PC com o qual foi construído o firmware, listado a seguir na Figura 2. 


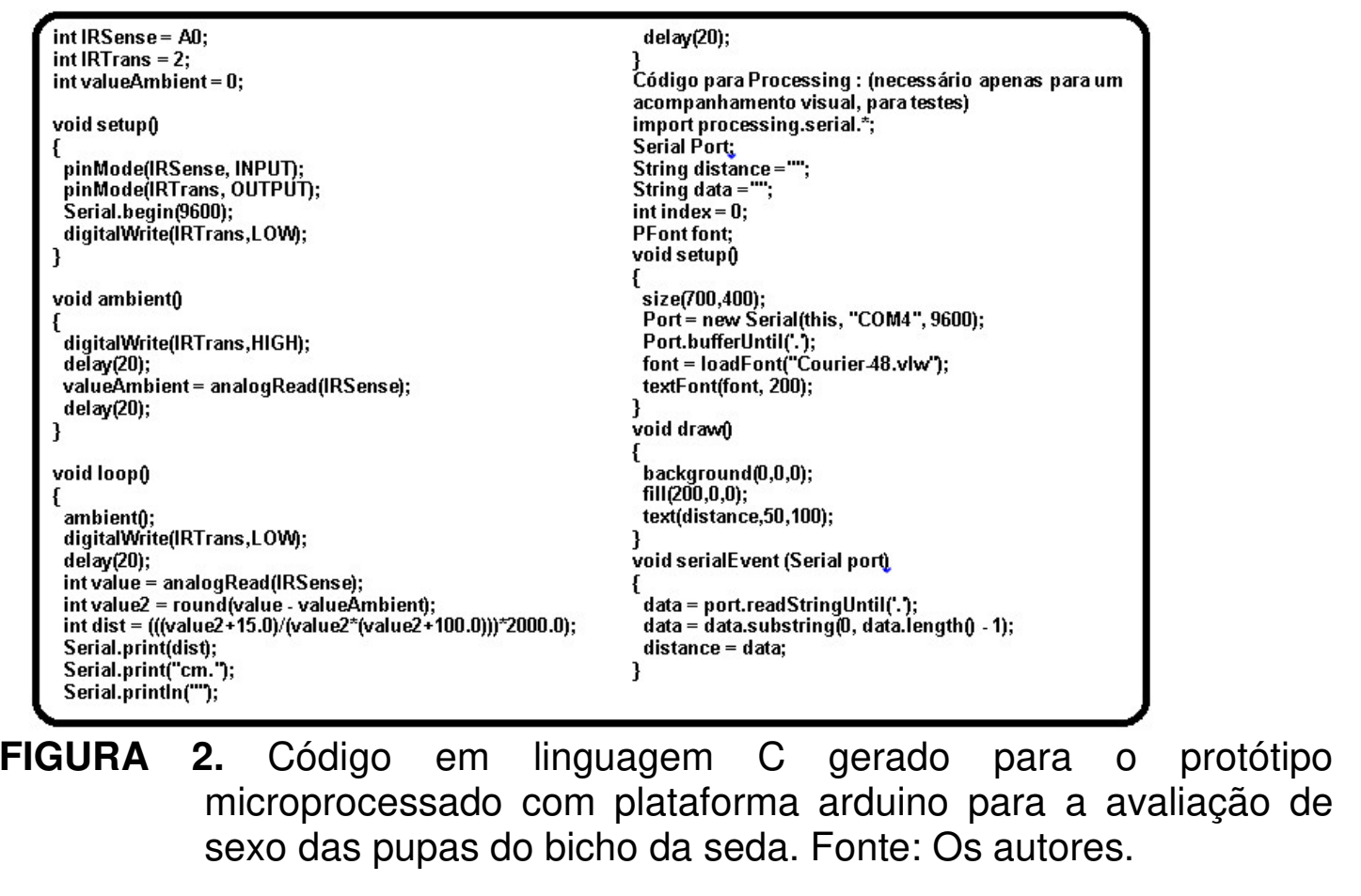

Os dados gerados pelas leituras automáticas realizadas pelo sistema com 0 firmware apresentado na Figura 2, permitiram a construção do arquivo compatível com o software WEKA, que é um arquivo texto, formatado de maneira a organizar os dados coletados, com seus atributos correspondentes aos valores medidos e pelo sexo da pupa em estudo determinado pelo especialista. Este arquivo recebe a extensão ".arff" e tem o aspecto apresentado na Figura 3.

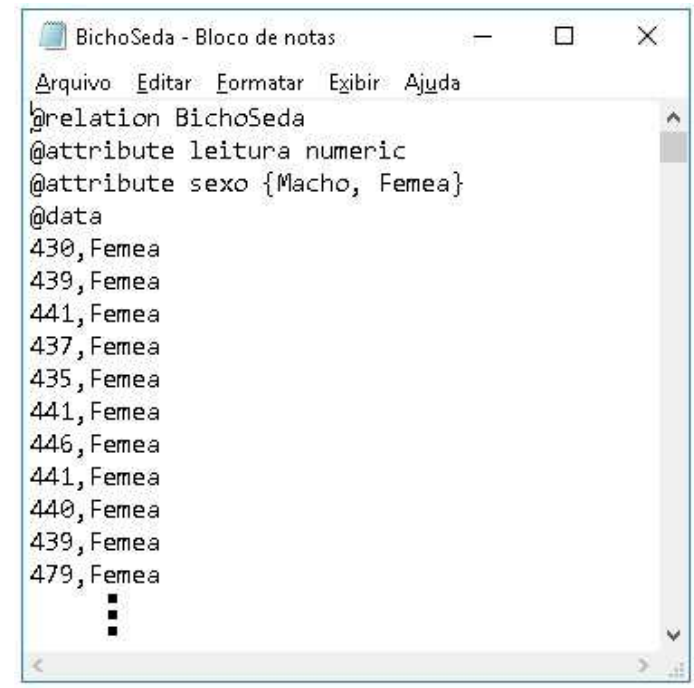

FIGURA 3. Arquivo ".arff" construído com os dados coletados no experimento para alimentação na ferramenta de mineração de dados WEKA. Fonte: Os autores.

Com o arquivo ".arff" apresentado na Figura 3, foi executado com o software WEKA o procedimento de classificação com a escolha do algorítmo J48, que que resultou na construção das saídas de respostas para a construção da árvore de ENCICLOPÉDIA BIOSFERA, Centro Científico Conhecer - Goiânia, v.14 n.26; p.942 
decisão. A tela do processamento correspondente pode ser visualizada na Figura 4.

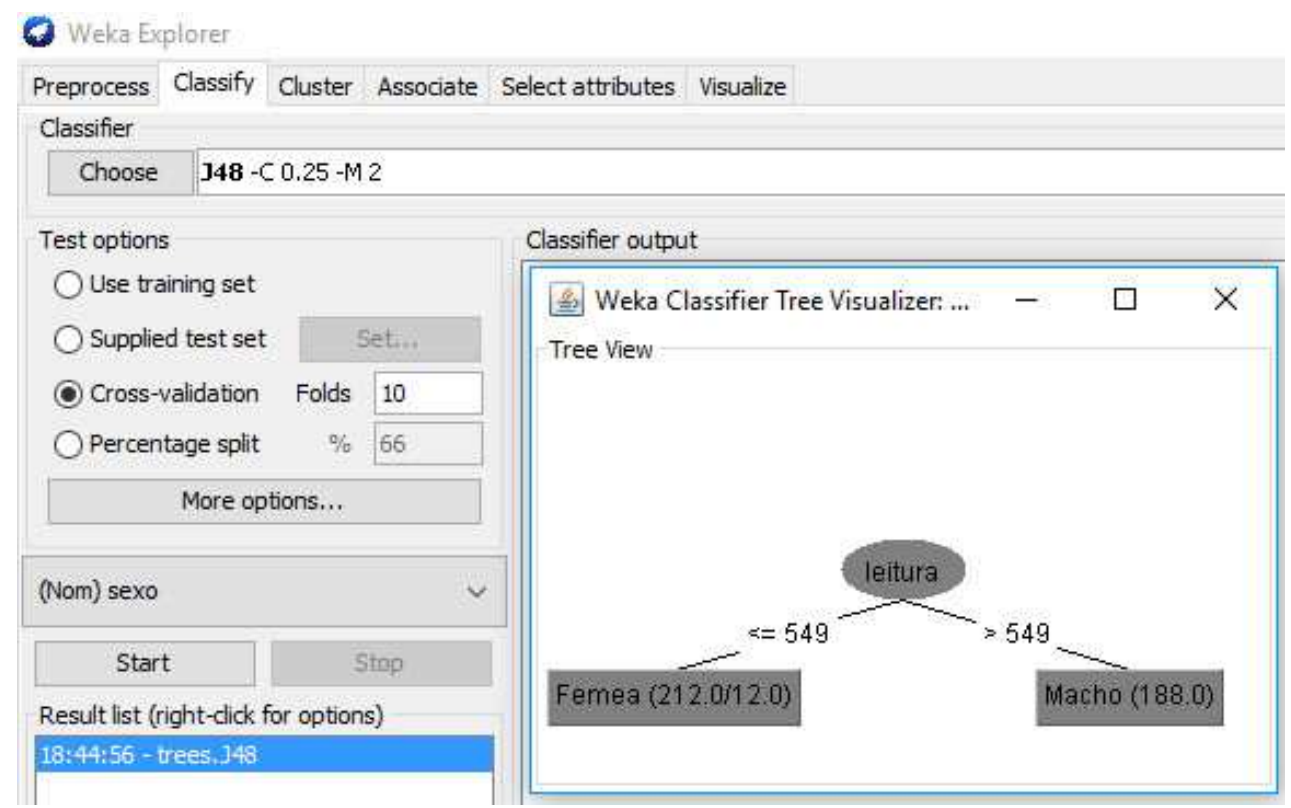

FIGURA 4. Tela de saída de resultados da ferramenta de mineração de dados WEKA com a obtenção da visualização da árvore de decisão gerada. Fonte: Os autores.

Como pode ser visto na Figura 4, a árvore de decisão separou um limiar em que, a quantidade de infravermelho que transpassou as pupas e que foi considerada para a tomada de decisão de identificação do sexo foi para o valores a seguir:

Se LEITURA maior que 549 (Bits) então o sexo é Masculino (ou Macho) e

Se LEITURA menor ou igual a 549 (Bits) então o sexo é Feminino (ou fêmea)

A Figura 5 exibe a tela do software WEKA que traz os resultados do algorítmo J48 com o sumário estatístico do processamento do conjunto de dados fornecido no arquivo "arff", onde é apresentada a quantidade de instância classificadas corretamente pelo algoritmo gerado. Como é possível observar na Figura 5, o algorítmo gerado tem uma capacidade de discriminação que permite classificar corretamente o sexo de pupas em até $96,75 \%$ das instâncias avaliadas e, incorretamente 3,25\%.

Este valor é muito mais alto do que os percentuais obtidos das inspeções visuais realizadas no processo produtivo convencional. Desta forma, o algorítmo foi portado para um sistema embarcado e o processo foi automatizado. Com base nos resultados obtidos, pode-se dizer que o desenvolvimento realizado permitiu a construção de um biossensor nas bases estudadas por Duhan et al. (2017) e Rehman et al. (2014), que trazem a idéia do uso dos novos Biosensores para aplicação a monitoramento no campo, e o mesmo configura-se como sendo uma aplicação de Agricultura de Precisão (AP), que dá o suporte necessário ao sucesso do empreendimento da criação do bicho da seda.

O processo utilizado, com a aplicação de um microcontrolador com um par emissor e receptor de infravermelho, com a coleta dos níveis de bits correspondentes à quantidade de infravermelho que transpassou o torax das pupas, ENCICLOPÉDIA BIOSFERA, Centro Científico Conhecer - Goiânia, v.14 n.26; p.943 
teve o processo de construção semelhante ao que pode ser visto nos trabalhos de Kamtongdee et al. (2013) e Sumriddetchkajorn et al. (2015) que aplicaram o mesmo método com sucesso em suas pesquisas.

Associando o tratamento destes dados coletados no experimento com a técnica da mineração de dados, nas bases adotadas na pesquisa de Naik e Samant (2016), e com a aplicação de classificação de dados, para gerar uma árvore de decisão, mostra que, superados os problemas na execução da AP com a interpretação dos dados coletados, obteve-se a compreensão durante a análise dos mesmos que é possível a geração de uma árvore de decisão com boa precisão de classificação. Como o bicho da seda é uma cultura específica, o sistema utilizado pode ser, também, considerado como uma estratégia sólida para a gestão da variabilidade de todo o processo produtivo como apresentado na pesquisa de Murakami et al. (2007).

O circuito microcontrolado foi desenvolvido com um módulo Arduino de baixo custo e sensores ópticos do tipo LED e Fototransistor, também de baixo custo, e escolhidos segundo as indicações da pesquisa de Ojha et al. (2015). Observa-se que o produto final gerado tem um ótimo custo benefício para uma aplicação voltada ao agronegócio, o que vai de encontro ao texto da pesquisa de Fisher e Kebede (2011), que dizem que há muitas vantagens no desenvolvimento de circuitos baseados em microcontroladores incorporando novas tecnologias de sensores em aplicações agrícolas de automação e tomada de decisão inteligente, para o monitoramento em tempo real.

A possibilidade alcançada com o equipamento desenvolvido, com a detecção das variáveis correspondentes ao número de bits que efetivamente transpassou o corpo das pupas para o processo, de uma forma não intrusiva, com nenhum ser humano interferindo diretamente, foi exatamente compatível com o previsto no trabalho de Mollo Neto (2007) e baseou-se na ideia de de poder extrair padrões ocultos ou informações implícitas previamente desconhecidas, sendo no caso desta pesquisa, o sexo das pupas do bicho da seda, à partir de grandes repositórios de dados, coletados das amostras do experimento, que foi a metodologia anteriormente apresentada por REZENDE et al. (2005).

No momento estão sendo preparados os testes de validação na fábrica para a futura implementação de um completo sistema automatico de detecção e classificação de sexo de pupas do bicho da seda (Bombyx Mori). 


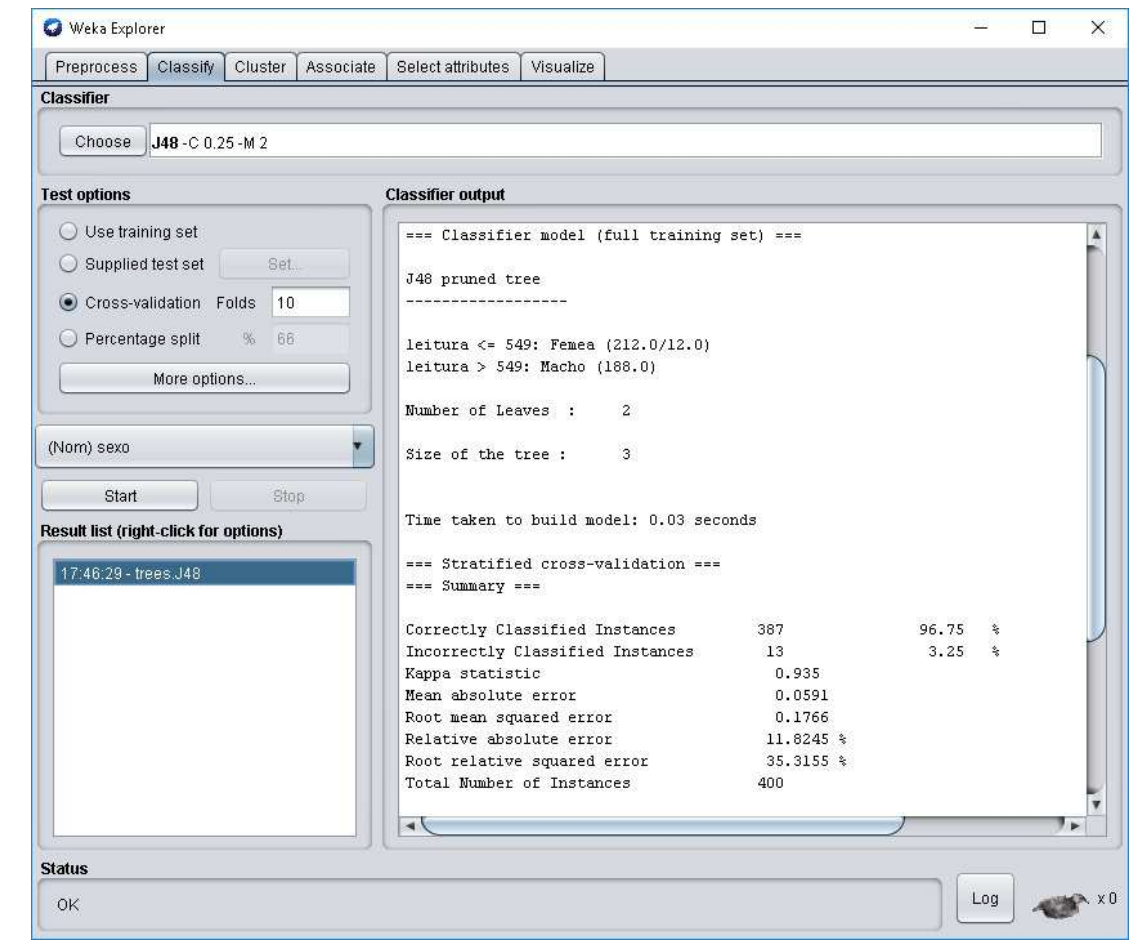

FIGURA 5. Resultados do algorítmo J48 com o sumário estatístico do processamento do conjunto de dados e resultados da precisão de classificação. Fonte: Os autores.

\section{CONCLUSÃO}

Foi possível concluir, que a utilização do processo de mineração de dados, voltado a classificação com a aplicação do algoritmo J48 permitiu obter um algoritmo que possibilita grande precisão na discriminação dos sexos das pupas do bicho da seda, auxiliando no processo produtivo que, atualmente, utiliza-se de meios manuais e lentos para este trabalho. O sistema automatizado agregará muito valor, com incremento de velocidade e redução de custos ao atual processo produtivo.

\section{AGRADECIMENTOS}

Os pesquisadores agradecem à UNESP, pelo uso das infraestruturas de pesquisa e dos laboratórios didáticos para os estudos e à bolsa PIBIC concedidas, assim como ao CNPq pela bolsa Produtividade DT.

\section{REFERÊNCIAS}

BRASIL. Ministério da Agricultura, Pecuária e Abastecimento. Agricultura de precisão Ministério da Agricultura, Pecuária e Abastecimento. Secretaria de Desenvolvimento Agropecuário e Cooperativismo. - Brasília: Mapa/ACS, 2011. Disponível em: <http://www.agricultura.gov.br/assuntos/insumosagropecuarios/arquivos-publicacoes-insumos/agric_precisao.pdf> Acesso em: 21/09/2017.

DUHAN,J. S.; KUMAR, R.; KUMAR, N.; KAUR, P.; NEHRA, K.; DUHAN, S. Nanotechnology: The new perspective in precision agriculture. Biotechnology Reports v. 15, p.11-23, 2017. Disponível em: <http://www.sciencedirect.com/science/article/pii/S2215017X16301400> Acesso em: 02/09/2017. DOI: https://doi.org/10.1016/j.btre.2017.03.002 
FISHER, D. K.; KEBEDE, H. A low-cost microcontroller-based system to monitor crop temperature and water status. Computers and Electronics in Agriculture, n.74 v.1 p. 168-173, 2010. Disponível em: <http://www.sciencedirect.com/science/article/pii/S0168169910001419> Acesso em: 21/09/2017. DOI: https://doi.org/10.1016/j.compag.2010.07.006

GAN, L.; WANG, Y.; XI, J.; NIU, Y.; QIN, H.; SIMA,Y.; XU, S. Long SAGE analysis of genes differentially expressed in the midgut and silk gland between the sexes of the silkworm Bombyx mori. African Journal of Biotechnology Vol. 11(49), pp. 1099411012, 19 June, $2012 . \quad$ Disponível em: < https://www.ajol.info/index.php/ajb/article/view/128689Acesso em: 21/09/2017. DOI: http://dx.doi.org/10.5897/AJB11.4121

KAMTONGDEE, C.; SUMRIDDETCHKAJORN, S.; SA-NGIAMSAK, C. Feasibility Study of silkworm pupa sex identification with pattern matching. Computers and Electronics in Agriculture v.95, p.31-37, 2013. Disponível em: <http://www.sciencedirect.com/science/article/pii/S0168169913000768> Acesso em: 21/09/2017. DOI: https://doi.org/10.1016/j.compag.2013.04.002

MARTÍN-RAMOS, P.; LOPES, M. J.; SILVA, M. M. L.; GOMES, P. E. B.; SILVA, P. S. P.; DOMINGUES, J. P. P.; SILVA, M. R. First exposure to Arduino through peercoaching: Impact on students' attitudes towards programming. Computers in Human Behavior. v.76, p.51-58, 2017. Disponível em:<http://www.sciencedirect.com/science/article/pii/S0747563217304193> Acesso em: 02/09/2017. DOI: https://doi.org/10.1016/j.chb.2017.07.007

MOLLO NETO, M. Decisões usando inteligência artificial. In: COSTA NETO, P. L. O. Decisões na Gestão da Qualidade. São Paulo: Blücher, 2007. Disponível em: < https://www.blucher.com.br/livro/detalhes/qualidade-e-competencia-nas-decisoes-

730> Acesso em: 22/09/2017. ISBN: 9788521204077

MURAKAMI, E.; SARAIVA, A. M.; RIBEIRO JUNIOR, L. C. M.; CUGNASCA, C. E.; HIRAKAWA, A. R.; CORREA, P. L. P. An infrastructure for the development of distributed service-oriented information systems for precision agriculture. Computers and Electronics in Agriculture, São Paulo, 2007. Disponível em: <http://www.sciencedirect.com/science/article/pii/S0168169907000609> Acesso em: 21/09/2017. DOI: https://doi.org/10.1016/j.compag.2006.12.010

NAIK, A.; SAMANT, L. Correlation Review of Classification Algorithm Using Data Mining Tool: WEKA, Rapidminer, Tanagra, Orange and Knime. Procedia Computer Science. $\quad$ v.85, p662-668, $2016 . \quad$ Disponível em:<http://www.sciencedirect.com/science/article/pii/S1877050916306019> Acesso em: 02/09/2017. DOI: https://doi.org/10.1016/j.procs.2016.05.251

OJHA, T.; MISRA, S.; RAGHUWANSHI, N. S. Wireless sensor networks for agriculture: The state-of-the-art in practice and future challenges. Computers and Electronics in Agriculture. v.118, p.66-84, $2015 . \quad$ Disponível em:<http://www.sciencedirect.com/science/article/pii/S0168169915002379> Acesso 
em: 02/09/2017. DOI: https://doi.org/10.1016/j.compag.2015.08.011

REHMAN, A.; ABBASI, A. Z.; ISLAM, N.; SHAIKH, Z. A. A review of wireless sensors and networks' applications in agriculture. Computer Standards \& Interfaces. v.36, n.2, p.263-270, $2014 . \quad$ Disponível em:<http://www.sciencedirect.com/science/article/pii/S0920548911000353> Acesso em: 02/09/2017. DOI: https://doi.org/10.1016/j.csi.2011.03.004

REZENDE, S.O.; PUGLIESI, J.B.; MELANDA, E.A.; DE PAULA, M.F. Mineração de dados. In: REZENDE, S.O. Sistemas inteligentes: fundamentos e aplicações. São Paulo: Manole, 2005. p.307-336. Disponível em: < https://www.saraiva.com.br/sistemas-inteligentes-fundamentos-e-aplicaes119681.html?pac_id=135884\&gclid=CjwKCAjwxo3OBRBpEiwAS7X62SX97b_0zOJc KceBx-0vA2K4p6SpmWrjdD8M-9ORqUdCMpn67aiohhoCAI8QAvD_BwE> Acesso em: 22/09/2017. ISBN: 8520416837

SUMRIDDETCHKAJORN, S.; KAMTONGDEE, C.; CHANHORM, S. Fault-tolerant optical-penetration-based silkworm gender identification. Computers and Electronics in Agriculture v.119, p.201-208, 2015. Disponível em: < http://www.sciencedirect.com/science/article/pii/S0168169915003051> Acesso em: 21/09/2017. DOI: https://doi.org/10.1016/j.compag.2015.10.004

TANG, X. D.; TU, Q.; MAO, F.; BAI, X.R. ; ZHUD, F. Characterization of an inhibitor of apoptosis gene (BmSurvivin-2) from the silkworm, Bombyx mori. Journal of AsiaPacific Entomology. v.20, n.4, p.1156-1160, 2017. Disponível em:<http://www.sciencedirect.com/science/article/pii/S1226861516305994> Acesso em: 02/09/2017. DOI: https://doi.org/10.1016/j.aspen.2017.08.027

YADAV, A. K.; CHANDEL, S. S. Solar energy potential assessment of western Himalayan Indian state of Himachal Pradesh using $\mathrm{J48}$ algorithm of WEKA in ANN based prediction model. Renewable Energy. v.75, p.675-693, 2015. Disponível em:<http://www.sciencedirect.com/science/article/pii/S0960148114006739> Acesso em: 02/09/2017. DOI: https://doi.org/10.1016/j.renene.2014.10.046 